\title{
Application of Comparators in Modern Power System Protection and Control
}

\author{
Ezechukwu O A. Phd, Mnse, Nieee \\ Department Of Electrical Engineering Nnamdi Azikiwe University Awka
}

\begin{abstract}
This paper presents the two basic comparison techniques- The amplitude and phase comparisons, used in power system protection and control. Phasor diagrams were used to discuss their relationships and circuit diagrams were modeled for the description of their operations. The general expression for impedance, Mho, positive offset and negative offset Mho characteristics were developed. At a stage simulation was done on a phase comparator to obtain the required characteristic.
\end{abstract}

Key words: amplitude comparison, Impedance characteristic, Phase comparator, Operating signal, Mho characteristic.

\section{Introduction}

A good protective relay must have, among others, good sensitivity, reliability and fast response. These qualities depend on the effectiveness of the comparator. Comparator, as the brain box of a relay, must recognize any change at the input terminals and react quickly. There are two methods of comparison: the amplitude and phase comparison techniques.

In amplitude comparison technique, the comparator produces an output whose amplitude is proportional to the amplitude difference of the input quantities; while in phase comparison technique, the comparator compares the phase angles of the input quantities and produces pulses whose width is proportional to the phase difference of the input quantities. The amplitude comparator can be used as phase comparator and vice versa, if certain modifications are made (see figs 1 and2)

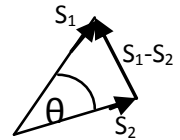

(a)

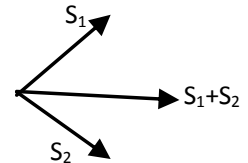

(b)

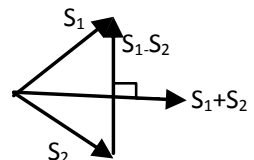

(C)

Fig1: analysis of the vectors, $\mathrm{S}_{1}$ and $\mathrm{S}_{2}$. (a)difference of two vectors [ amplitude comparison] (b) sum of 2 vectors and (c) combination of (a) and (b) [phase comparison].

Fig1, (a) shows the in put vectors of amplitude comparator, $S_{1}$ and $S_{2}$. (b) The sum of the vectors, $S_{1}$ and $S_{2}$ and (c) the combination of (a) and (b), which can be referred to as the phase comparison (amplitude comparison at $90^{\circ}$ criterion) with the inputs $\mathrm{S}_{1}-\mathrm{S}_{2}$ and $\mathrm{S}_{1}+\mathrm{S}_{2}$. So amplitude comparison can be equated to phase comparison at $\pm 90^{\circ}$ provided that the inputs to the phase comparator are:

$\mathrm{Sx}=\mathrm{S}_{1}-\mathrm{S}_{2} \quad$ (1a) and

$\mathrm{Sy}=\mathrm{S}_{1}+\mathrm{S}_{2} \quad(1 \mathrm{~b})$

Where Sx, the operating signal and Sy, the restraining signal, are the inputs to the phase comparator. At an angle of $90^{\circ}$, the operation of the phase comparator is marginal. When $\phi$ is more than $90^{\circ}$, the operation is completely restrained and when $\phi$ is less than $90^{\circ}$, the phase comparator produces an output which is the amplitude difference of the input signals See fig2. Therefore at $90^{\circ}$, an amplitude comparator can operate as phase comparator and vice versa. 


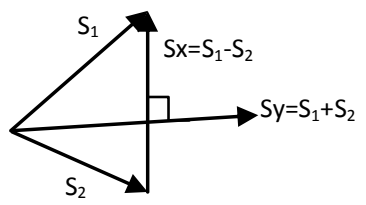

(a)

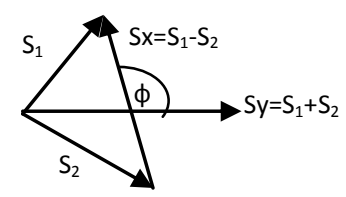

(b)

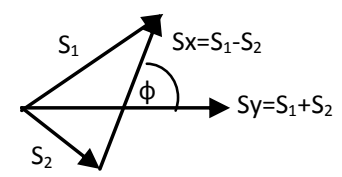

(C)

Fig2: modification of phase comparator. (a) $\phi=90^{\circ}, \mathrm{S}_{1}=\mathrm{S}_{2}, \mathrm{Sx}=0$; marginal operation

(b) $\phi>90^{\circ}, \mathrm{S}_{1}<\mathrm{S}_{2}$; operation restrained

(c) $\phi<90^{\circ}$. $S_{1}>S_{\text {, }}$ : operation enabled.

$\mathrm{S} 1$ is derived from current transformer and is shown as IZ in fig3 while $\mathrm{S} 2$ is derived from the voltage transformer and is shown as $\mathrm{V}$ in fig3.

Let the inputs to the amplitude comparator be;

$\mathrm{S} 1=\mathrm{IZ}$ (current converted to voltage)

And $\mathrm{S} 2=\mathrm{V}$

Such that using a phase comparator from equations (1) and (2) requires that:

$S_{x}=I Z-V$
$S_{y}=I Z+V$

The hardware necessary for realizing the phase comparator consists of readily available and well established small scale integrated circuits. Fig 3 is derived from the phasor diagram of fig 2.

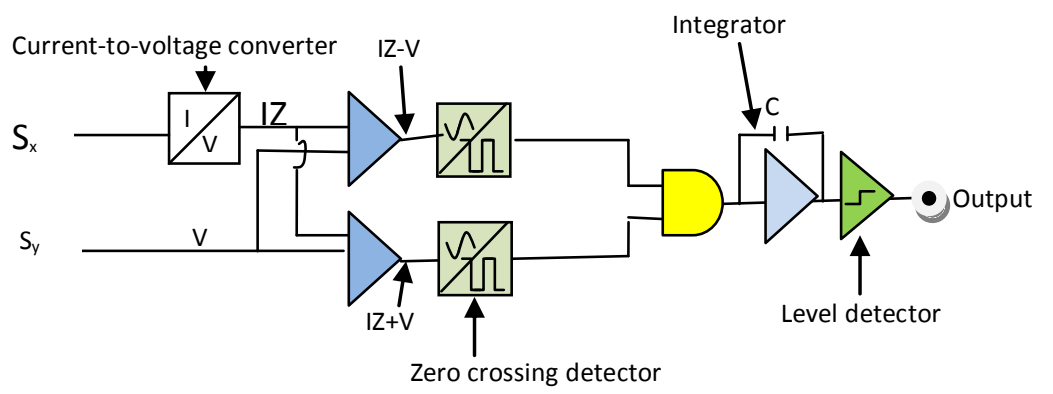

Fig3: one form of phase comparator to implement eqn (5).

From fig3, let the input to the comparator, $S x$ and Sy be IZ-V and IZ $+V$, respectively, such that at stable state: $|I Z-V|=|I Z+V|$ or $\left|A_{1} I Z+B_{1} V\right|=\left|A_{2} I Z-B_{2} V\right|$

Where; $\mathrm{A}_{1}, \mathrm{~A}_{2}, \mathrm{~B}_{1}$ and $\mathrm{B}_{2}$ are constants.

Dividing (6) by I; $\quad\left|A_{1} Z+\frac{B_{1} V}{I}\right|=\left|A_{2} Z-\frac{B_{2} V}{I}\right|$

If $\mathrm{A}_{1}=\mathrm{K}_{2}, \mathrm{~B}_{1} \mathrm{~V} / \mathrm{I}=\mathrm{K}_{1}, \mathrm{~A}_{2}=\mathrm{K}_{4}$ and $-\mathrm{B}_{2} \mathrm{~V} / \mathrm{I}=\mathrm{K}_{3}$, then eqn(7) becomes; $\left|K_{1}+K_{2} Z\right|=\left|K_{3}+K_{4} Z\right|$

Substituting $Z=R+j X$ in eqn [8],

$\mid\left(K_{1}+K_{2}(R+j X)|=| K_{3}+K_{4}(R+j X) \mid[9]\right.$

Then $\left|\left(K_{1}+K_{2} R\right)^{2}+\left(j K_{2} X\right)^{2}\right|=\left|\left(K_{3}+K_{4} R\right)^{2}+\left(j k_{4} X\right)^{2}\right|$

and $\left|\left(K_{1}+K_{2} R\right)^{2}+\left(j K_{2} X\right)^{2}\right|-\left|\left(K_{3}+K_{4} R\right)^{2}-\left(j k_{4} X\right)^{2}\right|=0$. Implying that;

$R^{2}-X^{2}+2 R \frac{\left(K_{1} K_{2}-K_{3} K_{4}\right.}{K_{2}^{2}-K_{4}^{2}}+\frac{K_{1}^{2}-K_{3}^{2}}{K_{2}^{2}-K_{4}^{2}}=0$

Comparing eqn [10] with the equation of a circle, $R^{2}+X^{2}+2 g R+2 h X+C=0$

Then; 
$g=\frac{K_{1} K_{2}-K_{3} K_{4}}{K_{2}^{2}-K_{4}^{2}}$

$h=0$ and $C=\frac{K_{1}^{2}-K_{3}^{2}}{K_{2}^{2}-K_{4}^{2}}$

So the characteristic is a circle on the $\mathrm{R}-\mathrm{X}$ diagram

With center

$\left(-\frac{g}{2}-\frac{h}{2}\right)$

When $\mathrm{K}_{1}=\mathrm{K}_{3}$, the radius $=\left(-\frac{g}{2}\right)$ and the circle passes through the origin.

When $K_{1} \neq K_{3}$, the circle becomes an offset envelop:

$K_{1}>K_{3}$, produces a positive offset while $K_{1}<K_{3}$, produces negative offset as shown in figs 8(c) and (d) respectively.

Fig3 shows one form of phase comparator which can be used in impedance measurement. It can therefore be used in distance protection. The simulated output is shown as fig 4 .

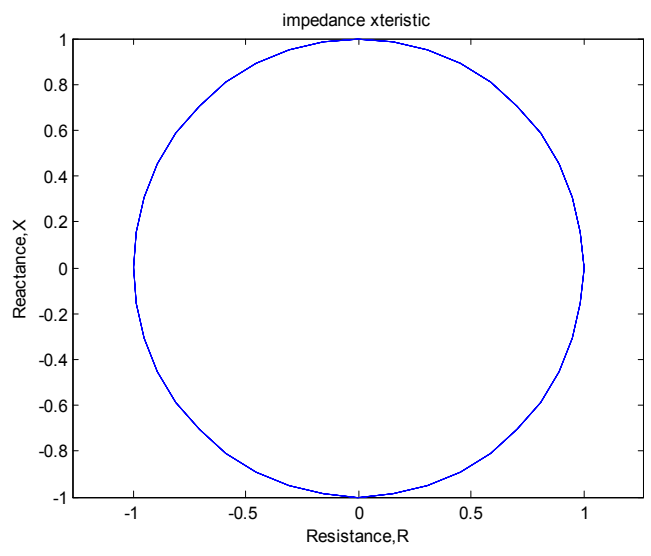

Fig4: Impedance characteristic simulated from fig3 at different angles

\section{Phase Comparison}

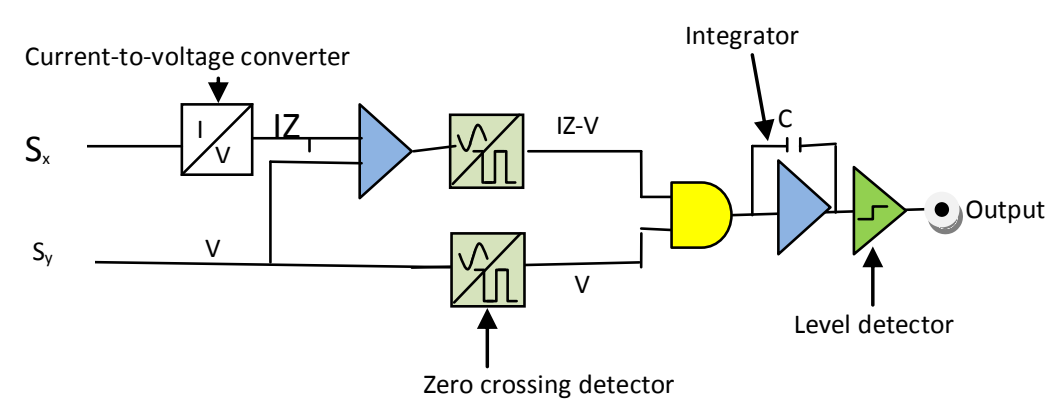

Fig 5 Phase comparator circuit for mho characteristic

A phase comparator which can be used to obtain mho characteristics can be developed from fig 2 if $\mathrm{S}_{\mathrm{x}}=\mathrm{S}_{1}-\mathrm{S}_{2}$ and $\mathrm{S}_{\mathrm{y}}=\mathrm{S} 2$. Where $\mathrm{S}_{1}=\mathrm{IZ}$ and $\mathrm{S}_{2}=\mathrm{V}$. One way of realizing this is shown in fig.5.

In this technique, the operating signal, Sx and the restraining signal Sy are fed into an AND gate used for coincidence detection. The width of the output signal from the coincidence detector is proportional to the phase difference of the two input signals. This output signal $|\mathrm{V}|$. $|\mathrm{IZ}-\mathrm{V}|$, can be fed into an integrator. The output 
of the integrator is fed to a level detector. The level detector can be set to correspond to any desired phase angle trip level.

In fig 5, the inputs to the coincidence detector are $\mathrm{K}_{1} \mathrm{~V}_{1}-\mathrm{K}_{2} \mathrm{IZ}$ and $\mathrm{K}_{3} \mathrm{~V}$

Where $\mathrm{K}_{1}, \mathrm{~K}_{2}$ and $\mathrm{K}_{3}$ are factors.

For stability;

$\left|K_{1} V_{1}-K_{2} I Z\right|=\left|K_{3} V\right|$

Dividing equation [11a] by I

$\left|\frac{K_{1} V-K_{2} Z}{I}\right|=\left|K_{3} \frac{V}{I}\right|$

If $\frac{V}{I}=Z_{K}$, eqn $[11 \mathrm{~b}]$ becomes

$\left|K_{1} Z_{K}-K_{2} Z / I\right|=\left|K_{3} Z_{K}\right|$

Now if $\mathrm{K}_{1} Z_{\mathrm{k}}=\mathrm{Z},\left|\frac{K_{2} Z}{I}\right|=\left|\frac{Z_{r}+Z_{0}}{2}\right|$ and $K_{3} Z_{k}=\frac{Z_{r}-Z_{0}}{2}$

Then eqn $[11 \mathrm{c}]$ becomes

$\left|Z-\frac{Z r+Z o}{2}\right|=\left|\frac{Z r-Z o}{2}\right|$

Now take $\mathrm{Z}=\mathrm{R} \pm \mathrm{JX}$ so that eqn [12] becomes

$\left|R+j X-\frac{\left[\left(R_{r}+j X_{r}\right)+\left(R_{o}+j X_{o}\right)\right]}{2}\right|$

$=\left|\frac{\left(R_{r}+j X_{r}\right)-\left(R_{o}+j X_{o}\right)}{2}\right| \equiv$

$\left[R-\left(\frac{R r+R_{0}}{2}\right)\right]^{2}+\left[X-\left(\frac{X r+X_{0}}{2}\right)\right]^{2}=\left(\frac{R r-R_{0}}{2}\right)^{2}+\left(\frac{X_{r}-X_{0}}{2}\right)^{2}$

$\mathrm{Rr}, \mathrm{Ro}, \mathrm{Xr}$ and Xo are values for particular characteristics, hence eqn [13] can be written in a more generalized form as

$(R-A)^{2}+(X-B)^{2}=C$

Where $\mathrm{A}, \mathrm{B}$ and $\mathrm{C}$ are given as follows;

$A=\frac{R r-R o}{2}$
$B=\frac{X r+X o}{2}$ and

$C=\left(\frac{R r-R o}{2}\right)^{2}+\left(\frac{X r-X o}{2}\right)^{2}$

Equation [13] is a general equation consisting of Mho, offset Mho, and impedance relay characteristics. Substituting the proper values of A, B and C, the appropriate characteristics can be derived.

When $\mathrm{Ro}_{\mathrm{o}}=\mathrm{Xo}_{\mathrm{o}}=0$, the Mho characteristics is derived and shown in fig.6(b). Consequently, equation [15] becomes

$$
\begin{aligned}
& A=\frac{R r}{2} \\
& B=\frac{X r}{2} \text { and } \\
& C=\left(\frac{R r}{2}\right)^{2}+\left(\frac{X r}{2}\right)^{2}
\end{aligned}
$$

For a positive offset Mho characteristics, $\mathrm{Ro}_{0}=-\mathrm{Ro}$ and $\mathrm{Xo}=-\mathrm{Xo}$. So that equation [15] becomes 


$$
\left.\begin{array}{l}
A=\left(\frac{R r+R 0}{2}\right) \\
B=\left(\frac{X r+X 0}{2}\right) \\
C=\left(\frac{R r+R 0}{2}\right)^{2}+\left(\frac{X r+X 0}{2}\right)^{2}
\end{array}\right\}
$$

For impedance characteristic, there is no displacement at the center of origin;

$\mathrm{Ro}=-\mathrm{Rr}$ and $\mathrm{Xo}=-\mathrm{Xr}$ in eqn [13], so that eqn [15] becomes

$\mathrm{A}=\mathrm{B}=0$ and

$$
C=R_{r}^{2}+X_{r}^{2}
$$

Therefore, the criteria for operation of impedance relay becomes

$$
R^{2}+X^{2} \leq R_{r}^{2}+X_{r}^{2}
$$

Therefore a Mho, offset Mho and impedance characteristics can be realized with equation [13] by substituting appropriate values of A,B, and C in equation [15]. Typical characteristics for impedance, Mho and offset Mho reler ${ }^{n}$ e shown in R-X plane in fig6 (a), (b), (c) and (d), respectively.

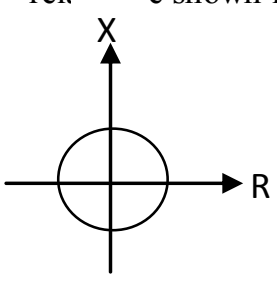

(a) Impedance characteristic

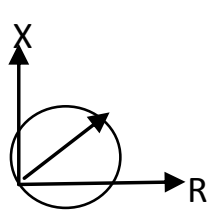

b) Mho characteristic

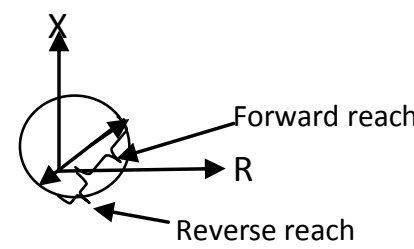

(c) Negative off-set Mho

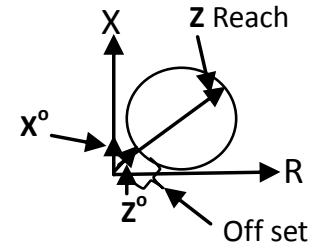

(d) Positive off- set Mho

Fig6:Characteristics for (a) Impedance relay (b) Mho relay ( c ) Negative offset Mho and (d) Positive offset Mho relay.

\section{III . Over/Under Voltage Protection}

Comparators are also used for voltage protection. One form of the protection circuit is shown in fig.7. The out put equation is $\mathrm{So}= \pm(\mathrm{V}-\mathrm{Vref}$.$) .$

Where $\mathrm{So}=$ Output signal,

$\mathrm{V}=$ The measurand and $\mathrm{Vref}=$ The reference voltage.

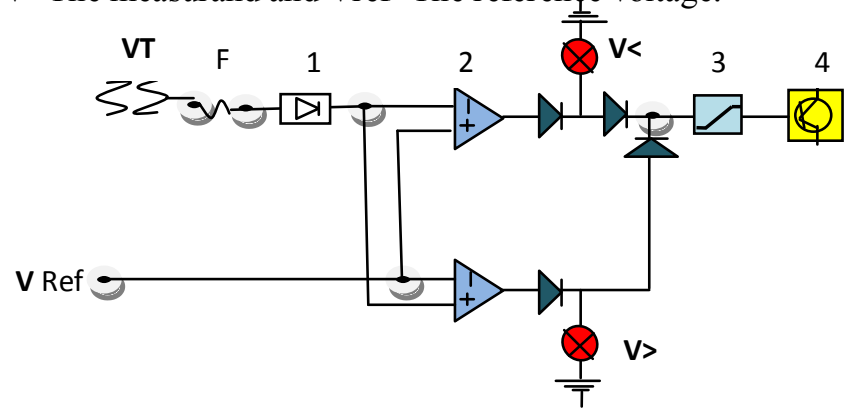

Fig 7: Basic circuit for over/under voltage protection

Eqn(19) yields zero output under normal voltage condition. When there is excess voltage, eqn(19) becomes V $>$-Vref And at low voltage it is Vref- $\mathrm{V}<$
KEY
VT-Voltage transformer
Vref-reference voltage
F-fuse
1. Full wave rectifier
2. Comparator
3. Level detector
4. Switching device

V> Overvoltage indication indication

V> Overvoltage indication indication 


\section{Conclusion}

Some applications of comparators in power system protection are presented. More applications can still be derived because there is no aspect of power system protection where comparators are not used. The choice between amplitude and phase comparator depends on the situation and convenience. The protection engineer has to decide.

\section{References}

[1]. Ezechukwu OA-The universal comparator UNIZIK Awka 2000

[2]. GEC-Protective relay application guide, GEC measurement PLC 1987.

[3]. Badri R, Vishwkarma DN- Power system protection and switch gear, Tata Mcgraw-Hill New Delhi 1995.

[4]. GEC.- The use of $\mathrm{R} \_X$ diagrams in relay work. GCE measurement PLC.

[5]. Reyrolle coy.- Operation and recommendations for type THR distance protection. Tech report No 611/or/311.

[6]. Ezechukwu OA and Anyanwu DL. Calibration report for GEC YTG distance relay. Benin 1981.

[7]. John AT- Generalized phase comparator for distance protection. IEE Power Record. IEE Savoy place, London. Vol119, pgs 833847 Sept 1972 